\title{
Uma abordagem heurística para a programação da produção na indústria de fundição com utilização da lógica fuzzy
}

\author{
A heuristic approach for production scheduling \\ in the foundry industry using fuzzy logic
}

\author{
Raul Landmann ${ }^{1}$ \\ Rolf Hermann Erdmann²
}

\begin{abstract}
Resumo: Este artigo descreve a concepção, desenvolvimento e aplicação de uma metodologia para a programação da produção na indústria de fundição. Há dois momentos importantes e interligados, que são a programação do forno e a programação das máquinas de moldagem. O problema consiste em determinar um sequenciamento adequado de ordens de produção nas linhas de moldagem, combinando peças leves com peças de peso médio e com peças pesadas, de modo a obter uma demanda constante de metal líquido, em equilíbrio com a oferta de metal proveniente do forno. Foi adotada a abordagem heurística para a solução, com utilização da lógica fuzzy, que oferece mecanismos para a representação e manipulação do conhecimento de especialistas, identificado em uma pesquisa qualitativa. Os resultados da aplicação demonstraram, além dos benefícios da sistematização do conhecimento e da capacidade de realizar simulações com muita rapidez, um desempenho mais eficiente que o obtido pelos profissionais da fundição.

Palavras-chave: Competitividade. Programação da produção. Heurística. Lógica fuzzy. Indústria de fundição.
\end{abstract}

\begin{abstract}
This article describes the conception, development, and application of a methodology for the production scheduling in the foundry industry. There are two important and related situations involved, which are the furnace scheduling and the molding machine scheduling. The problem consists in determining an adequate sequencing of production orders in the molding line combining light weight parts with medium and heavy parts in order to obtain a constant demand of molten metal balanced with the offer of furnace derived metal. The heuristic approach was chosen to find a solution to the problem, and using fuzzy logic technique, which allows modeling complex systems using a higher level of abstraction originating from knowledge and experience identified in a qualitative research. The results demonstrated, besides the benefits of the knowledge systematization and the capacity to run quick simulations in order to find the best alternatives to a solution, a more efficient performance than that obtained by the foundry professionals.
\end{abstract}

Keywords: Competitiveness. Production scheduling. Heuristic. Fuzzy logic. Foundry industry.

\section{Introdução}

Sacomano e Azzolini Júnior (2001) apontam que, já no final da década de 60, começava a sobressair a importância da manufatura dentro da estratégia organizacional, no tocante à contribuição para a sobrevivência, lucratividade e crescimento do empreendimento, a partir de um trabalho pioneiro desenvolvido por Skinner (1969). Dentro deste contexto, os sistemas de administração da produção passaram a desempenhar um papel cada vez mais importante para que a manufatura pudesse atingir os seus objetivos. A grande preocupação na gestão de sistemas de produção é melhorar o desempenho dos recursos produtivos em qualquer unidade econômica, agregando valor ao bem ou serviço produzido, quer seja por meio da melhoria da qualidade, aumento da produtividade, redução do custo ou diminuição do prazo de entrega, como expõe Machline (1994), para quem a perseguição simultânea desses quatro objetivos assegurará a razão de ser do negócio, o lucro sobre o investimento.

Nas empresas industriais, o Planejamento e Controle da Produção (PCP) têm, portanto, uma participação fundamental para a competitividade, com reflexos diretos e imediatos no custo e no

\footnotetext{
${ }^{1}$ Universidade da Região de Joinville - UNIVILLE, Campus Universitário - Rua Paulo Malschitzki, 10 - Zona Industrial, CEP 89219-710, Joinville - SC, Brasil, E-mail: raul.landmann@ univille.br

${ }^{2}$ Universidade Federal de Santa Catarina - UFSC, Campus Universitário, Trindade, CEP 88.040-900, Florianópolis - SC, Brasil,

E-mail: erdmann@cse.ufsc.br
} 
atendimento ao cliente. Na programação da produção, que se encontra no nível mais detalhado e complexo de um sistema de PCP, conforme salientam Terra e Pereira (2000), concentram-se importantes decisões de nível operacional, como a designação dos pedidos de clientes às máquinas, a liberação, o processamento e a expedição desses pedidos. Apesar disso, observa Saisse (2003), a programação da produção tem sido pouco tratada do ponto de vista estratégico, e a maior parte da literatura em estratégia de manufatura, em se tratando de sistemas de gerenciamento da produção, resume-se a um processo de escolha entre ferramentas clássicas como o MRP, kanban ou OPT.

Este trabalho focaliza o PCP, mais especificamente a atividade de programação da produção, na indústria de fundição. O segmento das fundições é bastante expressivo no País, tanto em termos tecnológicos como econômicos. Um levantamento realizado pela Associação Brasileira de Fundição em 1998 (ABIFA, 2004) detectou a existência no Brasil de 1.015 indústrias de fundição. Em 2003, havia 46.700 pessoas empregadas nas fundições existentes no Brasil, que produziram 2.083.581 $\mathrm{t}$ de fundidos $(86 \%$ correspondem a ferro fundido), tendo exportado $16 \%$ desse volume. Fernandes e Leite (2002) apontam que o Brasil posicionou-se, em 1997, como o $9^{\circ}$ produtor mundial de fundidos, segundo dados da revista Modern Casting.

A programação da produção numa fundição tem dois momentos importantes e interligados: a programação do forno (definição da liga a ser produzida em cada período) e a programação das máquinas de moldagem (definição dos moldes a serem produzidos). Este aspecto, salientado por Southall e Law (1980), é corroborado por Vianna e Arenales (1995) e Araújo e Clark (2001).

Existem várias soluções para problemas de programação da produção para fundições. Algumas técnicas procuram considerar as restrições relevantes para a geração dos programas que maximizem os objetivos de negócio colocados no momento para a empresa, tais como algoritmos genéticos, programação linear, heurística, regras de sequenciamento e outras.

O método algorítmico é um procedimento consistente e bem definido para resolver um problema. Trata-se, como afirmam Shimizu (1982) e DeMarco (1989) de uma abordagem que sempre garante uma boa solução. Os algoritmos determinísticos otimizantes, embora apresentem soluções ótimas, requerem um tempo computacional elevado. Além disso, como salienta Tubino (1997), soluções otimizantes, como, por exemplo, a clássica programação linear, embora viáveis matematicamente, na prática são de difícil aplicação devido à dificuldade de conciliar a variabilidade dos dados do sistema de produção com a dinâmica de atualização dos parâmetros do algoritmo.
A essência da abordagem heurística reside na aplicação de rotinas seletivas que reduzem a dimensão do problema e pode ser usada para simular o padrão de decisão dos seres humanos, mostrando-se adequada para os tipos de indústrias que possuem grande complexidade, diversidade e dinamismo em seus processos.

Foi detectada, tanto na revisão bibliográfica como na pesquisa de campo, a inexistência de uma técnica especificamente voltada para a geração de um programa de produção de curtíssimo prazo com um sequenciamento tal que promova o equilíbrio da capacidade de geração de metal na fusão com a demanda de metal absorvido na moldagem.

Optou-se pela abordagem heurística para a busca de uma solução. As regras de decisão dos especialistas da fundição foram identificadas por uma pesquisa qualitativa e, com a utilização dos conceitos da lógica fuzzy, que tem encontrado um campo fértil de aplicações, inclusive para o planejamento e controle da produção, foi desenvolvido um modelo para a programação da produção em fundições em que as operações de fusão e de moldagem ocorrem de forma simultânea.

Neste artigo, é apresentado o modelo heurístico criado com utilização da lógica fuzzy para sequenciar as ordens de produção na moldagem e gerar um programa de produção integrado com a fusão, no curtíssimo prazo, que reconhece inclusive os períodos de maior e menor disponibilidade de energia elétrica na indústria de fundição, e discutem-se os resultados obtidos com sua aplicação.

\section{A abordagem heurística e a inteligência artificial para a solução de problemas de programação da produção}

A palavra heurística deriva do verbo grego heuriskein, que significa "achar" ou "descobrir". Atualmente, a heurística é conceituada como "técnicas, em geral, que melhoram o desempenho de métodos de solução de problemas" observa Pöltl (2001). Para Gündra et al. (2002), métodos heurísticos são algoritmos que não fornecem necessariamente uma solução ótima, mas permitem chegar, por meio de cálculos, a uma solução aceitável, dentro de um período de tempo razoável. Segundo Fiedler, Greistorfer e Voss (2004), em geral pode-se entender a heurística como um método de solução de problemas que permite achar soluções boas com custos aceitáveis.

Sauer (1997) salienta que, a partir do início dos anos 80 , vêm sendo introduzidas novas técnicas de modelagem e de solução de problemas, por meio de abordagens por inteligência artificial, para suportar a solução de problemas práticos de sequenciamento. Autores como Fiedler, Greistorfer e Voss (2004) 
referem-se a elas como "meta-heurísticas", pois entendem que proporcionam uma estratégia superior, que geralmente orienta e modifica uma heurística simples aplicada a um problema específico, para a obtenção de uma solução melhor que esta heurística encontraria isoladamente.

A lógica fuzzy é uma técnica pertencente aos domínios da inteligência artificial, que é uma área baseada em várias disciplinas, como informática, biologia, psicologia, linguística, matemática e engenharia, cujo objetivo é desenvolver computadores que consigam pensar, ver, ouvir, andar, falar e sentir, destaca O’Brien (2001, p. 259).

\subsection{Lógica fuzzy}

A lógica fuzzy é, portanto, um método heurístico que utiliza a teoria de conjuntos fuzzy, integrando a área de inteligência artificial, no campo das aplicações da Ciência Cognitiva, juntamente com os algoritmos genéticos e as redes neurais (O’BRIEN, 2001). É também uma disciplina associada à construção e à programação de computadores para simular os processos de raciocínio humanos, salientam Heizer e Render (2001), que oferece mecanismos para a representação e manipulação do conhecimento de especialistas. Bonventi Jr. (1998) comenta que seu emprego é apropriado em determinadas condições, como existência de variáveis contínuas, sistema muito complexo para ser calculado em tempo real, e existência de especialistas que possam indicar regras de comportamento do sistema e os conjuntos difusos que representem a característica de cada variável.

A lógica fuzzy, explicam Heizer e Render (2001), permite trabalhar com valores aproximados, influências e dados incompletos ou ambíguos para tomar decisões. A capacidade de classificar de modo impreciso as variáveis de um problema, em termos de conceitos qualitativos, em vez de quantitativos, traduz a ideia de uma variável linguística, asseveram Almeida e Evsukoff (2003). Uma variável linguística é definida como uma entidade utilizada para representar de modo impreciso e, portanto, linguístico, um conceito ou uma variável de um determinado problema, admitindo como valores apenas expressões linguísticas, como "frio", "muito grande", "aproximadamente alto" etc. O processo de representação fuzzy de conhecimento depende fundamentalmente deste conceito.

Nobre (2000) expõe que o projeto e desenvolvimento de um sistema com a lógica fuzzy pode ser resumido em três etapas. O primeiro passo consiste em descrever o sistema por um conjunto de regras linguísticas ou frases de declarações do tipo se - então acrescentados dos conectivos lógicos and e or. As variáveis do sistema são definidos como variáveis linguísticas e assumem valores linguísticos ou palavras, não se restringindo a um valor numérico. Este tipo de declaração, conforme
Almeida e Evsukoff (2003) foi proposto na década de 70 por Mamdani, sendo conhecido como modelo de Mamdani, também chamado de inferência Max-Min, por utilizar as operações de união e de intersecção entre conjuntos por meio de operadores de máximo e de mínimo.

Após utilizar o conhecimento de especialistas para definir o conjunto de regras que irá compor a base do sistema, inicia-se o segundo passo, que consiste em definir as funções de pertinência que devem caracterizar quantitativamente os valores das variáveis linguísticas.

O terceiro e último passo consiste em definir o mecanismo de inferência para manipulação das regras e para a tomada de decisões, requerendo-se também a definição das interfaces de entrada e saída do sistema que utiliza a lógica fuzzy. A interface de entrada tem a função de mapear as informações numéricas do ambiente para valores caracterizados por funções de pertinência, transformando informações quantitativas em qualitativas (fuzzyfication). A interface de saída executa o mapeamento inverso à entrada (defuzzyfication), transformando informações qualitativas em quantitativas, quando convertida em valor escalar proporcional, ou transformando informações qualitativas em qualitativas para serem utilizadas diretamente em diagnóstico qualitativo de tomada de decisão.

\section{Procedimentos metodológicos}

A população pesquisada neste estudo é constituída por quatro fundições de ferro, mecanizadas, que apresentam processos com as características de fundição flow-shop. Todas têm expressiva participação no exigente mercado externo, e possuem avançada tecnologia de fabricação, com seus processos certificados por organismos internacionais de qualidade. Foram entrevistados em cada uma das empresas profissionais das áreas de PCP, logística e operações, do nível de direção ao de operação. Como procedimento para a coleta de dados foi utilizado o método de entrevistas estruturadas e não estruturadas, seguindo um roteiro previamente elaborado, bem como a observação sistemática e não participante.

Os dados coletados foram registrados nos roteiros utilizados, sistematizados e analisados com adoção do método da análise de conteúdo, tendo-se gerado relatórios descritivos para cada um dos casos pesquisados, que, após consolidados individualmente, permitiram gerar a síntese interpretativa de todos os casos, que por sua vez foi comparada com os fundamentos teóricos pesquisados.

$\mathrm{O}$ modelo foi criado a partir das constatações advindas das análises efetuadas, testado por simulações em planilha eletrônica, aplicado em uma das fundições que fez parte da população pesquisada, e os resultados interpretados e avaliados. 
O esquema geral do trabalho está delineado na Figura 1.

\subsection{Síntese dos resultados da pesquisa}

O sistema de PCP em todos os casos estudados apresenta como lógica básica o cálculo de recursos, o atendimento aos clientes como prioridade principal, a administração de restrições múltiplas (uso eficiente dos gargalos, com antecipação de ordens) e a utilização de procedimentos heurísticos para a programação de curto prazo.

Sistemas de planejamento da produção com capacidade finita disponíveis no mercado (Advanced Planning Systems - APS), assim como também desenvolvimentos próprios pelas fundições visitadas já proporcionam soluções para vários destes procedimentos. Todavia, para realizar a programação conjunta da fusão e da moldagem, foi observado que os programadores adotam unicamente regras empíricas advindas de seu conhecimento prático. Ficou evidenciada a ausência de uma sistemática que pudesse ser utilizada para esta finalidade. É um problema mal resolvido pelos especialistas em programação nestas fundições, agravado pela necessidade de reprogramações constantes no curto prazo, em decorrência da dinâmica do ambiente.

\section{Desenvolvimento do modelo e resultados obtidos}

A construção do modelo heurístico para o sequenciamento de ordens de produção para a programação conjunta da fusão e da moldagem, teve origem no diagnóstico elaborado a partir do estudo dos casos, amparado pela revisão de literatura.

Este procedimento, baseado na lógica fuzzy, está inserido na estrutura básica do sistema de PCP, e para desenvolver o modelo, que compreende a descrição do conjunto de regras linguísticas, a definição das funções de pertinência (valores das variáveis linguísticas), e o estabelecimento do mecanismo de inferência para manipulação das regras e para a tomada de decisões, com definição das interfaces de entrada e saída do sistema, foi seguido o roteiro apresentado por Nobre (2000).

A função deste modelo, implantado em uma planilha Excel com utilização de linguagem de macro Visual Basic (VBA), é sistematizar os procedimentos heurísticos utilizados pelas fundições para a programação da produção a curtíssimo prazo, simultaneamente para a fusão e a moldagem, e que se referem a regras para:

- Programar peças leves no horário de ponta (quando há restrição de energia) nas linhas de moldagem atendidas pela fusão; e

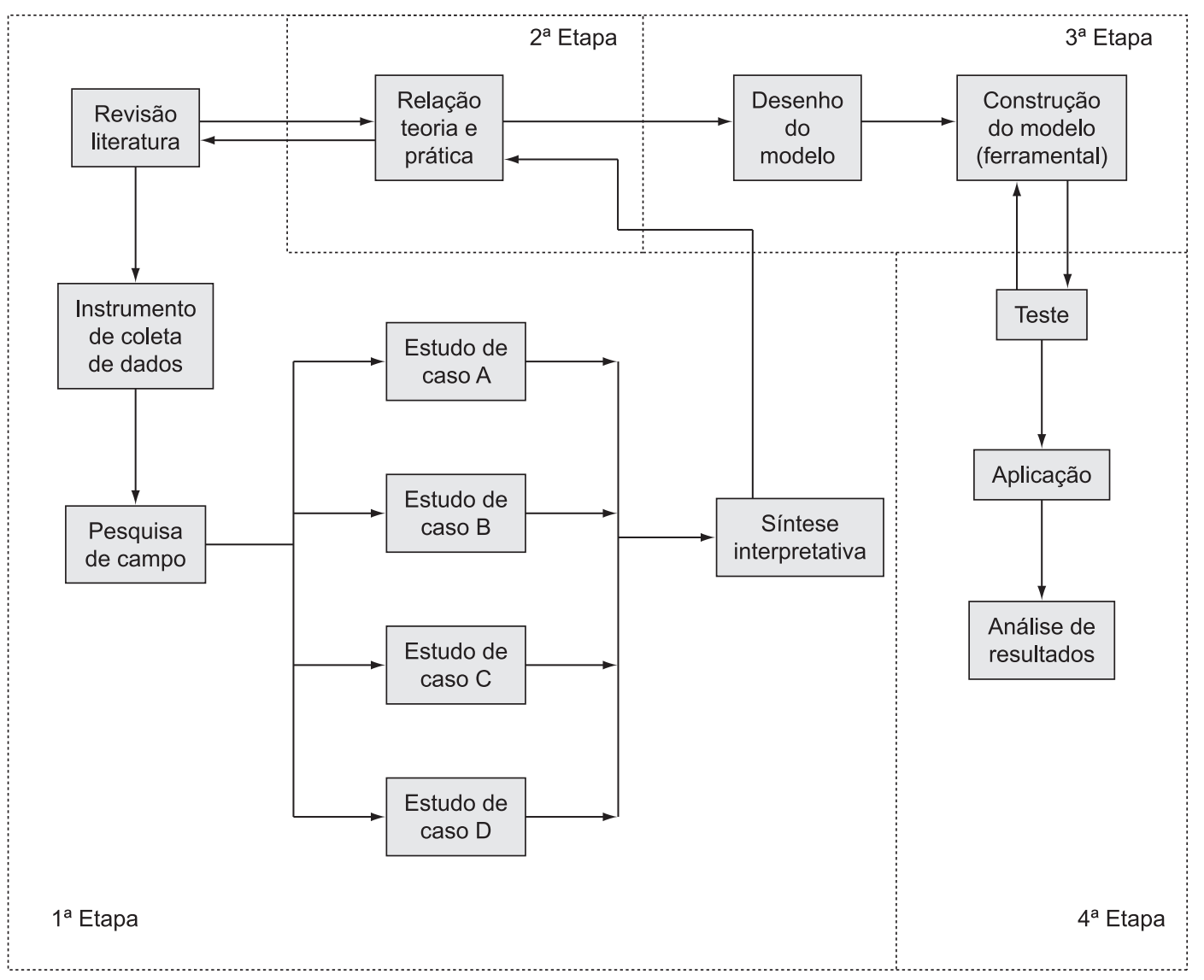

Figura 1. Representação esquemática da metodologia adotada. Fonte: Adaptado de Erdmann (1998) e Zattar (2004). 
- Programar uma combinação adequada de peças leves, peças de peso médio e peças pesadas nas linhas de moldagem fora do horário de ponta, de forma a obter uma demanda de metal líquido o mais constante possível ao longo de uma jornada de trabalho.

Neste trabalho, foram adotadas as seguintes convenções para caracterizar o peso das peças (no jargão dos fundidores, o peso da peça refere-se ao peso do conjunto peça fundida + canais + massalotes, também conhecido como "árvore"): $\mathrm{L}=$ leve (peças leves); $\mathrm{M}=$ média (peças de peso médio); $\mathrm{P}=$ pesada (peças pesadas).

\subsection{Construção do modelo}

A construção do modelo heurístico segundo a lógica fuzzy, utilizando os dados de uma das fundições visitadas, que possue uma unidade de fusão e três linhas de moldagem, compreendeu as seguintes etapas:

a) identificação e descrição da base de regras linguísticas para peças simultaneamente em produção nas três linhas de moldagem, determinada pelas alternativas de combinações utilizadas pelo programador de produção (especialista humano):

- se houver uma peça leve e uma média, então a terceira deverá ser pesada; ou

- se houver uma peça leve e uma média, então a terceira deverá ser média também; ou

- se houver uma peça leve e mais uma leve, então a terceira deverá ser pesada; ou

- se houver uma peça leve e uma pesada, então a terceira deverá ser pesada também; ou

- se houver uma peça média e mais uma média, então a terceira deverá ser média também; ou

- se houver uma peça média e uma pesada, então a terceira deverá ser média também.

As combinações que não forem compatíveis com estas regras configuram condições de rejeição. O Quadro 1 apresenta as combinações permitidas.

Outra regra incorporada ao modelo refere-se ao aspecto da redução da oferta de energia elétrica em determinado período do dia (horário de ponta). Se o forno utilizar este insumo energético, há uma redução na capacidade de fusão e, assim, deverão ser programadas as peças mais leves na moldagem. Dessa forma, foram geradas regras linguísticas para contemplar esta situação:

- Se o horário é de ponta, então as peças em produção devem ser leves;

- Se o horário é de ponta, e não houver peças leves, então as peças em produção devem ser médias; e

- Se o horário é de ponta, e não houver peças leves e nem médias, então as peças em produção devem ser pesadas. b) definição dos limites máximos e mínimos (Max-Min) dos conjuntos fuzzy para cada uma das três linhas de moldagem:

Os itens de cada linha foram divididos em três classes, de forma que cada conjunto ficou com a mesma quantidade de itens. Os itens foram ordenados de acordo com o peso da árvore (do menor para o maior e classificados em Leves (L), que correspondem portanto a 1/3 dos itens, Médias (M), que correspondem também a 1/3 dos itens, e Pesadas (P), que correspondem igualmente a $1 / 3$ dos itens de cada linha. Cada conjunto de itens é específico para determinada linha. Assim, cada linha tem seus próprios itens de peças leves, médias e pesadas. Os limites Max-Min para cada conjunto de cada linha estão demonstrados na Figura 2.

Quadro 1. Combinações permitidas de peças simultaneamente em produção nas linhas de moldagem.

\begin{tabular}{|cccc|}
\hline Linha 1 & Linha 2 & Linha 3 & Combinações \\
\hline L & M & P & Uma leve, uma \\
L & P & M & média e uma pesada \\
M & L & P & \\
M & P & L & \\
P & L & M & \\
P & M & L & \\
L & P & P & Uma leve e \\
P & L & P & duas pesadas \\
P & P & L & \\
M & M & P & Duas médias e \\
M & P & M & uma pesada \\
P & M & M & \\
L & M & M & Uma leve e \\
M & L & M & duas médias \\
M & M & L & \\
L & L & P & Duas leves e \\
L & P & L & uma pesada \\
P & L & L & \\
M & M & M & Três médias \\
\hline
\end{tabular}

Fonte: Landmann (2005).

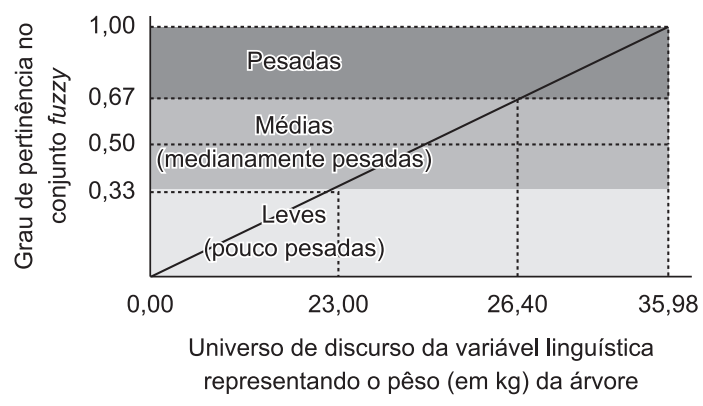

Figura 2. Funções de inferência fuzzy da linha I. Fonte: Landmann (2005). 
Para possibilitar um maior número de simulações na utilização do modelo, foram introduzidas mais duas opções de critérios para aplicar os limites:

- Ordenação por peso de todos os itens codificados nos diversos arquivos das linhas de moldagem, aplicando os indicadores fuzzy em função dos limites definidos, conjuntamente desde o item de menor peso de todas as linhas até o item de maior peso de todas as linhas;

- Realização da aplicação dos indicadores fuzzy em função dos limites definidos pelo peso dos itens em cada arquivo das linhas de moldagem isoladamente.

c) definição das interfaces de entrada e saída e criação do mecanismo de inferência:

Foram mapeadas as informações numéricas do ambiente para serem inseridas no sistema fuzzy do modelo heurístico (interface de entrada), constituídas por valores caracterizados pelas funções de pertinência, isto é, dentro dos limites Max-Min de cada conjunto nebuloso L (leve); M (média), P (pesada). Com isso, ocorreu a transformação de informações quantitativas em qualitativas (fuzzyfication).

Além dessas informações, por meio da interface de entrada é fornecido o programa de produção para um dia de 24 horas (itens e quantidades de moldes programados, ordenados por linha de moldagem em ordem de código de item) e outros dados, como:

- Código de cada item;

- Peso da árvore de cada item;

- Moldes/hora de cada linha; e

- $\mathrm{Kg} /$ hora demanda de metal.

O mecanismo de inferência, responsável pela combinação dos dados de entrada com as regras linguísticas, foi estabelecido por meio de heurísticas de factibilização, que são procedimentos definidos para manipular as regras e tomar decisões (SILVEIRA; MORABITO, 2002), tendo sido definidas aleatoriamente duas:

- Tipo 1: determina o sequenciamento do programa realizando uma ordenação crescente por peso dos itens das linhas que têm mais peças leves (do $\mathrm{L}$ para o M, e do M para o P) e uma ordenação decrescente por peso dos itens das linhas que possuem mais peças $\mathrm{P}$ (do $\mathrm{P}$ para o $\mathrm{M}$, e do $\mathrm{M}$ para o L). Se houver um número par de linhas de moldagem, em metade das linhas, é realizada a ordenação crescente dos itens pelo peso das peças (leve para pesado) e na outra metade das linhas a ordenação é feita de forma decrescente (pesado para leve). Se a quantidade de linhas de moldagem for ímpar, a ordenação crescente é realizada na metade das linhas menos 1 , e a outra metade das linhas é ordenada decrescentemente.
O pressuposto lógico que está por detrás deste procedimento do Tipo I é de que, na combinação das linhas, haverá um equilíbrio na demanda de metal, uma vez que algumas tiveram seus itens sequenciados, ao longo do tempo, dos itens leves aos pesados, e outras linhas, dos itens pesados aos leves.

- Tipo 2: escolhida por se assemelhar ao procedimento empírico adotado pelos especialistas da fundição, verifica a ocorrência de combinações não recomendadas (condições de rejeição) de itens simultaneamente em produção, em intervalos de 15 min ao longo de um dia de 24 horas (o intervalo de $15 \mathrm{~min}$ foi adotado por ser o tempo de set-up das linhas de moldagem). Identificada a condição de rejeição, o item que a provocou é remanejado para o final do dia, e os demais itens, em consequência, são antecipados. São feitas novas comparações com a base de regras, repetindo-se a operação até eliminar as condições de rejeição, ou acabarem as alternativas de remanejamento. Na hipótese da condição de rejeição se manifestar já no primeiro momento, a escolha do item a remanejar é aleatória, por meio de número randômico gerado pela planilha. A ordenação do Tipo 2, portanto, verifica a ocorrência de horários com condição indicativa de rejeição de acordo com as regras linguísticas. Verificada a ocorrência, o item programado que a causou é colocado no final da programação do dia na sua linha, ou seja, o último item naquela linha de moldagem. Após alteração no sequenciamento, é realizada nova verificação até eliminar todas as ocorrências ou acabarem as alternativas de troca.

A Figura 3 representa esta heurística.

A interface de saída faz o mapeamento inverso à entrada (defuzzyfication), e apresenta as informações qualitativas transformadas, tanto em qualitativas (sequenciamento resultante de peças Leves (L), Médias (M), e Pesadas (P)), como em quantitativas (peso de metal demandado pelas três linhas de moldagem, ao longo de um dia de $24 \mathrm{~h}$, a partir da quantidade de moldes programados para cada item, em cada linha de moldagem). O resultado é apresentado em planilha, cujos dados são transferidos para uma tabela na forma como são visualizados pelos especialistas da fundição (Tabela 1). A decisão é obter um sequenciamento tal que se obtenha o melhor equilíbrio entre a demanda de metal e a absorção pelas linhas de moldagem.

Em função da subjetividade do trabalho do especialista de PCP na fundição para sequenciar as ordens de produção do programa de moldagem, não foram identificados na pesquisa indicadores de 
desempenho para avaliar o sequenciamento gerado para o programa conjunto da fusão com a moldagem. Dessa forma, foram criados indicadores para avaliar as diferentes alternativas de simulação geradas pelo modelo e comparar os resultados, inclusive com o sequenciamento determinado pelo profissional da fundição:

- Desvio padrão em relação à média do peso total demandado;

- Quantidade de set-ups durante um dia de $24 \mathrm{~h}$;

- Tempo em que prevaleceu uma condição de rejeição não resolvida durante um dia de trabalho; e

- Capacidade de produção perdida no recurso gargalo ao longo de uma jornada.

Com todas essas implementações, o modelo heurístico para a programação conjunta da fusão e da moldagem, baseado na lógica fuzzy, apresenta a estrutura mostrada na Figura 4.

\subsection{Aplicação prática e resultados do modelo proposto}

A aplicação prática do modelo consistiu na realização do sequenciamento de itens durante vários dias de programação de uma das fundições pesquisadas. Assim, as necessidades foram processadas na planilha com o modelo proposto, utilizando as três opções de limites fuzzy para cada uma das heurísticas de factibilização (tipo 1 e tipo 2) do mecanismo de inferência, tendo sido gerado um programa de produção para cada uma destas alternativas. Cada alternativa teve seu desempenho mensurado pelos indicadores que foram definidos para avaliar o modelo.

Também o programa gerado empíricamente pelo programador de produção (especialista da fundição) foi avaliado frente aos mesmos indicadores.

São discutidos os resultados de quatro dias de aplicação do modelo e, para um dos dias, é apresentado o programa sequenciado pelo especialista da fundição e o mesmo programa gerado pela melhor alternativa do modelo proposto.

No dia 1, os resultados foram muito semelhantes em todas as alternativas analisadas. As simulações realizadas com a heurística de factibilização do tipo 1, com aplicação geral e aplicação isolada por linha dos limites fuzzy aparentemente são melhores por apresentarem um menor desvio padrão. A perda de capacidade foi de $5 \%$, para uma perda mínima admissível de $1,23 \%$ (média de $22.717 \mathrm{Kg}$ para uma capacidade de $23.000 \mathrm{Kg}$ na fusão).

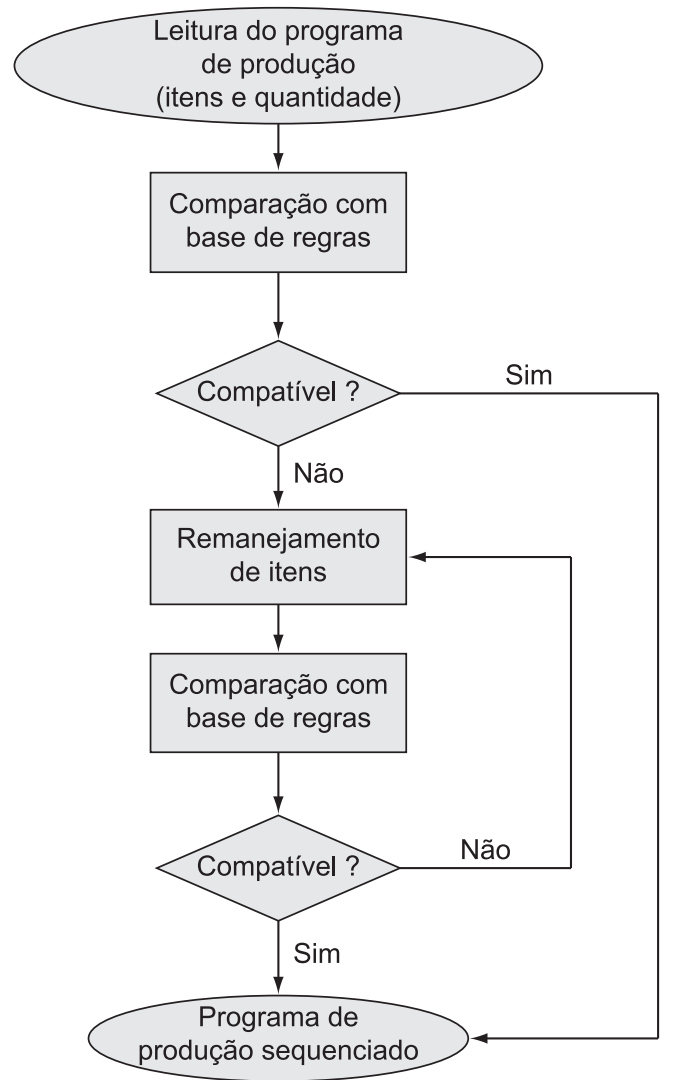

Figura 3. Heurística de factibilização do tipo 2. Fonte: Landmann (2005).

Tabela 1. Sequenciamento do programa de produção obtido em heurística baseada na lógica fuzzy.

\begin{tabular}{cccccc}
\hline \multicolumn{7}{c}{ Linha I } & \multicolumn{5}{c}{ Programação do dia (sequenciada) } \\
\hline Capac (mol/hora) & $\mathbf{3 0 0}$ & Capac (mol/hora) & $\mathbf{2 7 0}$ & Capac (mol/hora) & 250 \\
\hline Código & Quant (mol.) & Código & Quant (mol.) & Código & Quant (mol.) \\
\hline 122 & 430 & 208 & 1060 & 315 & 1000 \\
126 & 4300 & 220 & 1600 & 329 & 1100 \\
101 & 600 & 229 & 1300 & 331 & 500 \\
186 & 470 & 244 & 1200 & 332 & 600 \\
185 & 1060 & 245 & 700 & 348 & 1000 \\
- & - & - & - & 351 & 900 \\
- & - & - & - & 306 & 500 \\
\hline
\end{tabular}


No dia 2, mais uma vez, os desempenhos foram muito semelhantes, tendo a alternativa do tipo 1 , com aplicação dos limites fuzzy aplicados isoladamente por linha, apresentado o menor desvio padrão e a menor perda de capacidade (7\% para uma perda admissível de $5,85 \%)$. O programa gerado pelo especialista também apresentou a menor perda de capacidade (7\% para uma perda admissível de 4,85\%). A alternativa com tipo 1 e aplicação dos limites medianos apresentou a menor condição de rejeição, mas teve um maior desvio padrão e uma maior perda de capacidade.

No terceiro dia, a alternativa do tipo 1 com aplicação dos limites isolados por linha apresentou o menor desvio padrão, a menor condição de rejeição e a menor perda de capacidade (5\% para uma perda admissível de $0,7 \%$ ). O programa gerado pelo especialista teve uma perda de capacidade de $6 \%$. Isso significa que o modelo heurístico proporcionou um ganho de $1 \%$ na utilização da capacidade de fusão.

Para melhor ilustrar a aplicação do modelo heurístico, referente ao dia 4 serão apresentadas, além da planilha detalhada dos resultados gerados, o programa gerado pelo especialista (Tabela 2), e o programa gerado pela melhor simulação do modelo heurístico, que é a alternativa do tipo 2 com aplicação dos limites medianos (Tabela 3 ).

Tabela 2. Programa do dia 4 com sequenciamento elaborado pelo especialista.

\begin{tabular}{cccccc}
\hline \multicolumn{7}{c}{ Programa do dia 4 - Especialista } \\
\hline Linha 1 & \multicolumn{2}{c}{ Linha 2 } & \multicolumn{2}{c}{ Linha 3 } \\
\hline Capac (mol/hora) & $\mathbf{3 0 0}$ & Capac (mol/hora) & $\mathbf{2 7 0}$ & Capac (mol/hora) & $\mathbf{2 5 0}$ \\
\hline Código & Quant (mol.) & Código & Quant (mol.) & Código & Quant (mol.) \\
\hline 126 & 4000 & 253 & 1000 & 310 & 820 \\
128 & 1000 & 218 & 1300 & 313 & 700 \\
170 & 1000 & 219 & 1300 & 312 & 1200 \\
186 & 1300 & 251 & 400 & 329 & 2000 \\
- & - & 250 & 2000 & 351 & 1000 \\
- & - & - & - & 332 & 600 \\
\hline
\end{tabular}

Fonte: Landmann (2005).

Tabela 3. Programa do dia 4 com sequenciamento elaborado pelo modelo heurístico (melhor alternativa).

\begin{tabular}{cccccc}
\hline \multicolumn{7}{c}{ Programa do dia 4 - Modelo Heurístico } \\
\hline Linha 1 & \multicolumn{3}{c}{ Linha 2 } & \multicolumn{2}{c}{ Linha 3 } \\
\hline Capac (mol/hora) & $\mathbf{3 0 0}$ & Capac (mol/hora) & $\mathbf{2 7 0}$ & Capac (mol/hora) & $\mathbf{2 5 0}$ \\
\hline Código & Quant (mol.) & Código & Quant (mol.) & Código & Quant (mol.) \\
\hline 126 & 4000 & 218 & 1300 & 310 & 820 \\
128 & 1000 & 219 & 1300 & 312 & 1200 \\
186 & 1300 & 251 & 400 & 313 & 700 \\
170 & 1000 & 253 & 1000 & 351 & 1000 \\
- & - & 250 & 2000 & 329 & 2000 \\
- & - & - & - & 332 & 600 \\
\hline
\end{tabular}

Fonte: Landmann (2005).

Tabela 4. Indicadores do programa do dia 4.

\begin{tabular}{|c|c|c|c|c|c|}
\hline $\begin{array}{c}\text { Resultados do } \\
\text { sequenciamento }\end{array}$ & $\begin{array}{c}\text { Média } \\
(\text { Kg) }\end{array}$ & $\begin{array}{c}\text { Desvio padrão } \\
(\mathrm{Kg})\end{array}$ & $\begin{array}{l}\text { Número de } \\
\text { set-up }\end{array}$ & $\begin{array}{c}\text { Condição de } \\
\text { rejeição (minuto) }\end{array}$ & $\begin{array}{c}\text { Perda } \\
\text { capacidade }(\%)\end{array}$ \\
\hline \multicolumn{6}{|l|}{ Tipo I } \\
\hline Limite geral & 20.919 & 1.649 & 15 & 0 & 9 \\
\hline Limite isolado & 21.274 & 1.430 & 15 & 0 & 9 \\
\hline Limite mediano & 21.382 & 1.319 & 15 & 0 & 9 \\
\hline \multicolumn{6}{|l|}{ Tipo II } \\
\hline Limite geral & 21.277 & 3.268 & 15 & 225 & 11 \\
\hline Limite isolado & 21.470 & 3.378 & 15 & 180 & 11 \\
\hline Limite mediano & 21.692 & 2.590 & 15 & 45 & 8 \\
\hline Especialista & 21.073 & 2.144 & 15 & - & 10 \\
\hline
\end{tabular}

Fonte: Landmann (2005). 


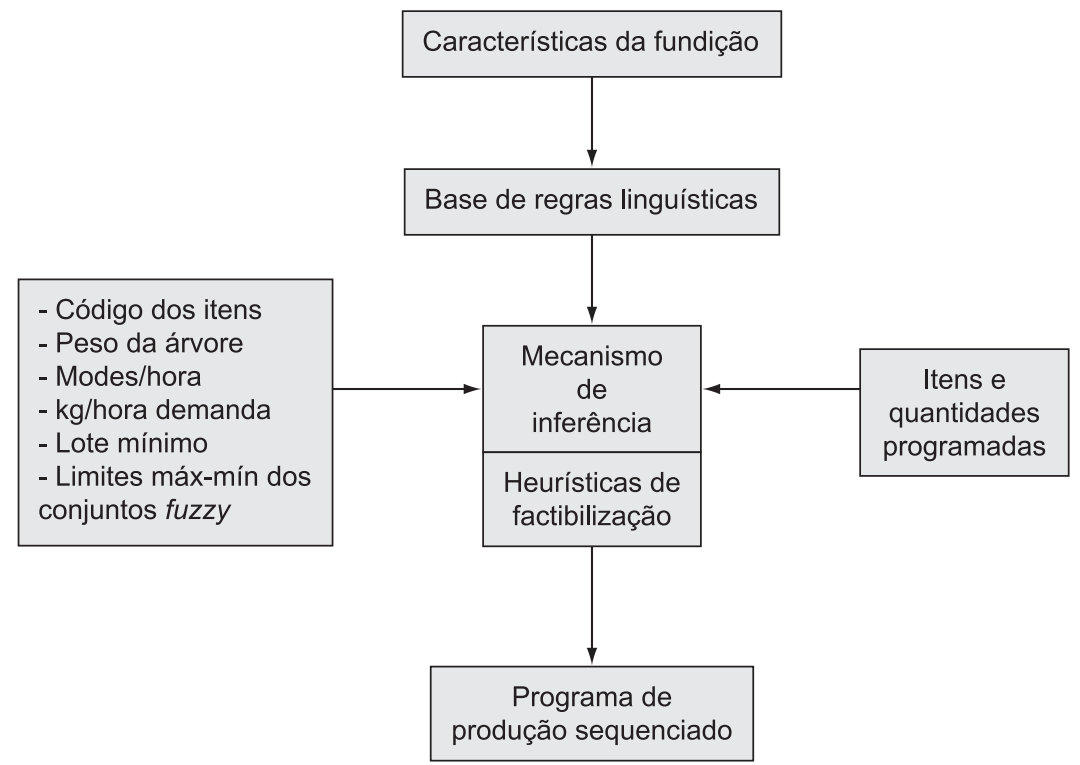

Figura 4. Estrutura do modelo heurístico baseado na lógica fuzzy para a programação conjunta do forno e das linhas de moldagem. Fonte: Landmann (2005).

Os resultados da simulação do dia 4 estão retratados na Tabela 4.

Neste dia, a melhor alternativa em termos de perda de capacidade ficou por conta da alternativa do tipo 2 com aplicação dos limites medianos. A perda foi de $8 \%$ (para uma perda admissível de 5,7\%), contra uma perda de $10 \%$ do programa gerado pelo especialista. Este ganho de $2 \%$ representa $11.040 \mathrm{Kg}$ de metal por dia, o que corresponde a $220.800 \mathrm{Kg}$ por mês, equivalentes a 9,6 horas adicionais de produção por mês. As alternativas do tipo 1 apresentaram um menor desvio padrão e uma menor condição de rejeição, mas uma maior perda de capacidade.

Os resultados da aplicação prática do modelo nestes quatro dias permitem afirmar que o modelo heurístico proposto conseguiu apreender bem as regras e os processos de raciocínio dos especialistas, pois em nenhum momento o programa gerado pelo modelo apresentou resultados inferiores aos do programa gerado pelo especialista, pelo contrário, em duas oportunidades (dias 3 e 4) o programa gerado pelo modelo heurístico apresentou resultados superiores, em termos de aproveitamento da capacidade de produção.

A geração de um programa de produção com um sequenciamento tal que permita, além de atender os pedidos nos prazos previstos, aproveitar bem a capacidade existente, atende perfeitamente as necessidades de qualidade e produtividade de uma fundição. Uma fundição que opera com menos interrupções minimiza as perdas de energia, pois o metal líquido no forno precisa ser mantido aquecido, o que significa manter o forno ligado, independentemente de estar ou não atendendo as linhas de moldagem. Além disso, os panelões de transporte de metal também precisam estar aquecidos, o que é favorecido com uma operação o mais contínua possível, o que contribui também para reduzir defeitos de fundição.

Percebe-se também que, dependendo das características do programa diário, diferentes alternativas podem gerar os resultados mais satisfatórios. Isso significa que, para cada dia, é conveniente simular todas as alternativas de sequenciamento, e selecionar aquela que melhor se adequa às necessidades momentâneas, produzindo os melhores resultados. As simulações na planilha eletrônica são executadas com muita rapidez e seus resultados podem ser analisados praticamente em tempo real. Este aspecto proporciona ao modelo um benefício adicional, no que se refere à análise de reprogramações que ocorre com frequência a qualquer momento do dia.

\section{Considerações finais}

Embora o ponto chave do PCP de uma fundição resida na programação conjunta e integrada da fusão e da moldagem, constatou-se, tanto na revisão da literatura como na pesquisa de campo, que este problema era resolvido empiricamente por meio do conhecimento prático e da experiência dos programadores de produção, inexistindo uma metodologia específica para tal fim. Uma programação adequada da fusão e da moldagem permite aproveitar melhor a capacidade de produção, bem como reduzir o desperdício de energia e os defeitos de fundição.

Assim sendo, a proposta deste trabalho foi desenvolver um modelo heurístico para a programação simultânea e integrada da fusão e da moldagem, inserido no sistema de PCP, em fundições mecanizadas. O modelo foi desenvolvido com base nos conceitos da 
lógica fuzzy, utilizando as regras de decisão utilizadas pelos programadores de produção, identificadas na pesquisa. Ele permite orientar o sequenciamento das ordens de produção na moldagem de forma a propiciar o melhor aproveitamento da capacidade produtiva, sem prejudicar o atendimento dos pedidos nas datas requeridas, já que seu horizonte de atuação se limita à programação diária.

Os resultados práticos da programação realizada durante alguns dias com o modelo heurístico desenvolvido demonstraram um desempenho equivalente, em alguns dias, e superior em outros, ao programa gerado pelo especialista da fundição. Outrossim, a possibilidade de transferência do conhecimento especializado para uma ferramenta gerencial é um aspecto muito importante a ser ressaltado com o desenvolvimento do modelo, pois propicia meios para o compartilhamento de informações e a aprendizagem organizacional.

Outra vantagem do método é a capacidade de permitir a realização de simulações em planilha eletrônica com extrema rapidez, permitindo analisar alternativas e selecionar as de melhor desempenho praticamente em tempo real. Esta característica é particularmente importante para análise de reprogramações que ocorrem durante o dia.

Há potencial para melhorar ainda mais o desempenho do modelo. A sua evolução poderia incluir a fragmentação de lotes nos períodos fora do horário de ponta, com o intuito de promover uma maior constância na demanda de metal líquido. Assim, novas versões do modelo poderiam considerar também regras adicionais e uma heurística de factibilização para a fragmentação de lotes. Isso acarretaria a inclusão do controle de tempos perdidos para set-up em linhas que não dispõem do chamado sistema de troca rápida, e da introdução do conceito de lote mínimo. Outro estudo, que poderia ser levado a efeito, se refere à ampliação dos conjuntos fuzzy. A existência de apenas três (peças leves, peças de peso médio e peças pesadas) promove uma amplitude muito grande no universo da variável linguística "peso", o que causa sensíveis variações na demanda de metal mesmo com as combinações permitidas. Assim, poderiam ser introduzidos conjuntos adicionais, do tipo "muito leve", "pouco leve", "muito pesada", "pouco pesada" etc.

Um campo altamente promissor que está sendo objeto de vários estudos recentes está ligado à computação evolutiva, cujos conceitos poderiam ser incorporados ao modelo. De acordo com Back (1996), trata-se de sistemas também chamados de algoritmos evolutivos, ou de algoritmos genéticos, para a resolução de problemas que utilizam modelos computacionais, baseados na teoria da evolução natural. Este campo de estudo apresenta um significativo potencial, inclusive com o desenvolvimento de modelos híbridos com utilização da lógica fuzzy associada aos algoritmos genéticos.

\section{Referências}

ALMEIDA, P. E. M.; EVSUKOFF, A. G. Sistemas fuzzy. In: REZENDE, S. O. (Org.). Sistemas inteligentes fundamentos e aplicações. São Paulo: Manole, 2003.

ARAÚJO, S. A.; CLARK, A. R. Um problema de programação da produção numa fundição. In: SIMPÓSIO BRASILEIRO DE PESQUISA OPERACIONAL SOBRAPO, 33., 2001, Campos do Jordão. Anais...

ASSOCIAÇÃO BRASILEIRA DE FUNDIÇÃO - ABIFA. Índices do mercado. 2004. Disponível em: <http:// www.abifa.org.br>. Acesso em: 25 jan. 2004.

BACK, T. Evolutionary algorithms in theory and practice. Oxford University Press, 1996.

BONVENTI Jr., W. Lógica "fuzzy": fundamentos e aplicabilidade. Revista Estudos Universitários, v. 24, n. 1, supl. 1, p. 125-146, 1998.

DEMARCO, T. Análise estruturada e especificação de sistemas. Rio de Janeiro: Campus, 1989.

ERDMANN, R. H. Organização de sistemas de produção. Florianópolis: Insular, 1998.

FERNANDES, F. C. F.; LEITE, R. B. Automação industrial e sistemas informatizados de gestão da produção em fundições de mercado. São Carlos. Gestão \& Produção, v. 9, n. 3, p. 313-344, 2002.

FIEDLER, C.; GREISTORFER, P.; VOSS, S. Metaheuristiken als moderne Lösungskonzepte für komplexe Optimierungsprobleme. Disponível em: <http://kfuniqnez.ac.at>. Acesso em: 15 fev. 2004.

GÜNDRA, H. et al. Heuristische Ansätze zur Lösung von Standortfragen un deren Implementierung. 2002. Disponível em: <http://www.itwm.fraunhofer. de>. Acesso em: 12 fev. 2004.

HEIZER, J.; RENDER, B. Administração de operações. 5. ed. Rio de Janeiro: Livros Técnicos e Científicos, 2001.

LANDMANN, R. Um modelo heurístico para a programação da produção em fundições com utilização da Lógica Fuzzy. Tese de Doutorado. Programa de Pós-Graduação em Engenharia de Produção. Universidade Federal de Santa Catarina, 2005.

MACHLINE, C. Evolução da administração da produção no Brasil. Revista de Administração de Empresas, v. 34, n. 3, p. 91-101, 1994.

NOBRE, F. S. M. Fuzzy logic: aplicações. s.l. Revista Integração, v. 6, n. 23, p. 264-267, 2000.

O'BRIEN, J. A. Sistemas de informação e as decisões gerenciais na era da internet. São Paulo: Saraiva, 2001.

PÖLTL, A. Scheduling durch Heuristische Suche - das ISIS-System. 2001. Disponível em: <http://informatik. uni-ulm.de. Acesso em 15 fev. 2004.

SACOMANO, J. B.; AZZOLINI Jr., W. Uma análise da evolução histórica da estrutura funcional do planejamento e controle da produção. In: ENCONTRO NACIONAL DE ENGENHARIA DE PRODUÇÃO, 21., 2001, Salvador. Anais... Salvador: ABEPRO, 2001.

SAISSE, M. C. Planejamento fino da produção - um elo esquecido na estratégia de manufatura. In: ENCONTRO 
NACIONAL DE ENGENHARIA DE PRODUÇÃO, 21. 2003, Ouro Preto. Anais... Ouro Preto: ABEPRO, 2003.

SAUER, J. Ablaufplanung (scheduling). 1997. Disponível em: <http://www-is.informatik.uni-oldenburg.de>. Acesso em: 15 fev. 2004.

SHIMIZU, T. Processamento de dados - conceitos básicos. São Paulo: Atlas, 1982.

SILVEIRA, R. R.; MORABITO, R. Um método heurístico baseado em programação dinâmica para o problema de corte bidimensional guilhotinado restrito. São Carlos. Gestão \& Produção, v. 9, n. 1, p. 78-92, 2002.

SKINNER, W. Manufacturing - the missing link in corporate strategy. Harvard Business Review, p. 12-17, 1969.

SOUTHALL, J. T.; LAW, T. D. Approaches to improved job scheduling in foundries. The British Foundrymen, p. 287-291, 1980.
TERRA, A. R. T.; PEREIRA, N. A. Aplicação de redes neurais artificiais na programação da produção. In: ENCONTRO NACIONAL DE ENGENHARIA DE PRODUÇÃO, 20., 2000, São Paulo. Anais... São Paulo: ABEPRO, 2000.

TUBINO, D. F. Manual de planejamento e controle da produção. São Paulo: Atlas, 1997.

VIANNA, A. C. G.; ARENALES, M. Um problema de programação da produção numa fundição automatizada.. São Carlos. Gestão \& Produção, v. 2, n. 3, p. 244-263, 1995.

ZATTAR, I. C. Análise da aplicação dos sistemas baseados no conceito de capacidade finita nos diversos níveis da administração da manufatura através de estudos de casos. 2004. 136 f. Dissertação (Mestrado em Engenharia Mecânica)-Universidade Federal de Santa Catarina, 2004. 